\title{
A pseudodifferential equation with damping for one-way wave propagation in inhomogeneous acoustic media
}

\author{
Christiaan C. Stolk* \\ Centre de Mathématiques, Ecole Polytechnique, 91128 Palaiseau Cedex, France
}

Received 29 September 2003; accepted 25 November 2003

Available online 27 February 2004

\begin{abstract}
A one-way wave equation is an evolution equation in one of the space directions that describes (approximately) a wave field. The exact wave field is approximated in a high frequency, microlocal sense. Here we derive the pseudodifferential one-way wave equation for an inhomogeneous acoustic medium using a known factorization argument. We give explicitly the two highest order terms, that are necessary for approximating the solution. A wave front (singularity) whose propagation velocity has non-zero component in the special direction is correctly described. The equation cannot describe singularities propagating along turning rays, i.e. rays along which the velocity component in the special direction changes sign. We show that incorrectly propagated singularities are suppressed if a suitable dissipative term is added to the equation.
\end{abstract}

() 2004 Elsevier B.V. All rights reserved.

MSC: $35 \mathrm{~L} 05 ; 35 \mathrm{~S} 10$

Keywords: One-way wave equation; Acoustic equation; Pseudodifferential calculus

\section{Introduction}

In this paper we consider one-way wave equations for inhomogeneous acoustic media in $n$ dimensions, where $n \geq 2$. We assume there is a special space direction, which we call depth or the vertical direction, with coordinate denoted by $z$, the other directions are called lateral or horizontal and are denoted by $x$. The time coordinate is denoted by $t$. The medium is described by its slowness (inverse velocity) $v=v(z, x)$ and its mass density $\rho=\rho(z, x)$. Let $U=$ $U(z, x, t)$ denote the acoustic wave field, and $F=F(z, x, t)$ a volume source, then the acoustic equation is given by

$$
\left(-\rho^{-1} v^{2} \partial_{t}^{2}+\sum_{j=1}^{n-1} \partial_{x_{j}} \rho^{-1} \partial_{x_{j}}+\partial_{z} \rho^{-1} \partial_{z}\right) U=F .
$$

A one-way wave equation is an equation describing only downward propagating waves, whose propagation velocity has positive vertical component, or only upward propagating waves, with negative vertical component of propagation velocity. As we will discuss here, by solving a one-way equation by progressing in depth in one direction

* Tel.: +33-1-69334594; fax: +33-1-69333019.

E-mail address: stolk@math.polytechnique.fr (C.C. Stolk). 
an approximation to (part of the) wave field is obtained. One-way equations are used in applications in geophysics (see, e.g. [1]) and ocean acoustics, for which many different numerical methods have been developed. References and a discussion of some of these can be found in, e.g. [2,4].

In an inhomogeneous medium reflections occur for waves with wavelength comparable to the scale of the medium variations. Only the high-frequency part can be expected to be computed using a method progressing in one direction only. However, this is not necessarily a disadvantage. In seismic imaging and migration an asymptotic limit is implicit, and the absence of reflections is often an advantage.

The high-frequency (or singular) part of solutions to wave equations is very well understood. High frequency waves propagate along rays, curved trajectories in space. We use the theory of microlocal analysis about this (see, e.g. the books $[3,6,9])$. Thus we consider approximation of the wave field modulo an error that is $C^{\infty}$, hence in Fourier space goes to zero when the frequency goes to infinity, faster than any negative power of the frequency. The singularities of the function, that is the part that does not go to zero rapidly when the frequency becomes large, can be localized in position and direction using the wave front set of Hörmander (see the mentioned references or [5]). For propagating singularities the position and direction determine the ray. Using these ideas we describe precisely how the wave field is approximated by solving a one-way wave equation.

Singularities propagating with velocity that is not horizontal can be described by a pseudodifferential evolution equation in $z$. Such equations are obtained from a factorization or decoupling argument, see, e.g. [8] or the similar treatment in [9, Section 9.1], or [6, Section 23.2]. However, such equations are not defined at the point where the ray is tangent to horizontal. Moreover, it is clear that waves propagated along turning rays cannot be computed by progressing in one direction in depth only. In practice it is desirable that such wave fronts are suppressed.

In this paper we give a pseudodifferential evolution equation in $z$ that includes such a suppression. Thus we obtain a model for numerical one-way wave equation methods. We give sufficient detail so that the equation can in principle be used as a starting point for discretization.

Our one-way wave equation is obtained in two steps. First we extend the factorization argument of Taylor [8]. We compute the pseudodifferential equation referred to above to the two highest orders that are at least needed for a highest order approximation of the solution (for such a pseudodifferential equation, as for the ordinary wave equation, the leading order term describes the behavior of the rays, while the next term describes the amplitudes to highest order). We also allow for a normalization in the definition of the down- and upward propagating parts of the wave field. With a suitably chosen normalization the equation becomes unitary, microlocally. We then modify this equation in order to suppress any wave fronts propagating along turning rays. We show that the solutions to the one-way equation approximate microlocally the real solution.

The precise formulation of these results is the subject of the next section. Sections 3 and 4 contain the proofs of two theorems.

\section{Pseudodifferential one-way wave equation and approximation of the solutions}

We first introduce some notation. The Fourier variables corresponding to $x, z$ and $t$ will be denoted by $\xi, \zeta$ and $\tau$, with Fourier transform defined by

$$
\hat{f}(\xi, \tau)=\iint \mathrm{e}^{-\mathrm{i}(\xi x+\tau t)} f(x, t) \mathrm{d} x \mathrm{~d} t .
$$

A function $\psi=\psi(x, t, \xi, \tau)$ in $C^{\infty}\left(\mathbb{R}^{n} \times \mathbb{R}^{n}\right)$ is a symbol of order $m$, if there are constants $C_{\alpha, \beta}$ such that

$$
\left|\partial_{x, t}^{\alpha} \partial_{\xi, \tau}^{\beta} \psi(x, t, \xi, \tau)\right|<C_{\alpha, \beta}(1+\|(\xi, \tau)\|)^{m-|\beta|}
$$

(see a text on pseudodifferential operators). Here $\alpha, \beta$ are a multi-indices $\alpha=\left(\alpha_{1}, \ldots, \alpha_{n}\right), \alpha_{j} \in \mathbb{Z}_{\geq 0},|\alpha|=\alpha_{1}+$ $\cdots+\alpha_{n}$. Associated with such a symbol $\psi$ is a pseudodifferential operator, that will be denoted by $\psi\left(x, t, D_{x}, D_{t}\right)$, and is given by 


$$
\psi\left(x, t, D_{x}, D_{t}\right) f=(2 \pi)^{-n} \int \psi(x, t, \xi, \tau) \hat{f}(\xi, \tau) \mathrm{e}^{\mathrm{i}(\xi x+\tau t)} \mathrm{d} \xi \mathrm{d} \tau .
$$

The set of symbols is denoted by $S^{m}\left(\mathbb{R}^{n} \times \mathbb{R}^{n}\right)$. We will typically encounter operators acting in $(x, t)$, depending on $z$, but independent of $t$ (of convolution type in $t$ ), i.e. with symbols $\psi(z, x, \xi, \tau)$. A symbol is in $S^{-\infty}$ if for any $N$ there is a constant $C$ such that $\left|\partial_{x, t}^{\alpha} \partial_{\xi, \tau}^{\beta} \psi\right|<C(1+\|(\xi, \tau)\|)^{-N}$ The corresponding operator then maps distributions to $C^{\infty}$. We will write $\psi_{1} \sim \psi_{2}$ if $\psi_{1}-\psi_{2} \in S^{-\infty}$.

We recall that, if $f(y), y \in \mathbb{R}^{n}$ is a distribution, Hörmander's wave front set $\mathrm{WF}(f)$ contains points $(y, \eta)$, contained in the cotangent space or phase space $\mathbb{R}^{n} \times \mathbb{R}^{n}$, that give positions and directions associated with the singularities of $f$, see [3, Section 1.3], or [5, Section 8.1]. This set is conic, i.e. if $(y, \eta) \in \operatorname{WF}(f)$ then $(y, \lambda \eta) \in \operatorname{WF}(f)$ for all $\lambda>0$. If $\Gamma$ is a conic subset of $\mathbb{R}^{n} \times \mathbb{R}^{n}$ we say that $f \equiv g$ microlocally on $\Gamma$ if $\operatorname{WF}(f-g) \cap \Gamma=\emptyset$.

Denoted by $p(z, x, \zeta, \xi, \tau)=\rho(z, x)^{-1} v(z, x)^{2} \tau^{2}-\rho^{-1}\left(\xi^{2}+\zeta^{2}\right)$ the principal symbol of $P$. If $U$ satisfies $P U=0$, then the singularities of $U$ are in the characteristic set given by

$$
p(z, x, \zeta, \xi, \tau)=0 .
$$

They propagate along null bicharacteristics, curves in the cotangent space contained in the set given by (5) that are solutions to the Hamilton vector field of $p$ (see [9, Theorem 6.2.1] or [6, Section 23.1]). Parameterizing by time, the differential equations for the null bicharacteristics are

$$
\begin{aligned}
& \frac{\mathrm{d} x}{\mathrm{~d} t}=-v(z, x)^{-2} \tau^{-1} \xi, \quad \frac{\mathrm{d} z}{\mathrm{~d} t}=-v(z, x)^{-2} \tau^{-1} \zeta, \\
& \frac{\mathrm{d} \xi}{\mathrm{d} t}=-\tau \nu^{-1} \frac{\partial \nu}{\partial x}, \quad \frac{\mathrm{d} \zeta}{\mathrm{d} t}=-\tau \nu^{-1} \frac{\partial \nu}{\partial z} .
\end{aligned}
$$

So singularities with $-\zeta / \tau>0$, satisfy $\mathrm{d} z / \mathrm{d} t>0$ (downgoing) and waves with $-\zeta / \tau<0$ satisfy $\mathrm{d} z / \mathrm{d} t<0$ (upgoing).

If a point $(z, x, \xi, \tau)$ is given with $\|\xi\|<v(z, x)|\tau|$, then there are two solutions $\zeta$ to (5). These will be denoted by $\pm b= \pm b(z, x, \xi, \tau)$, where

$$
b=-\tau \nu \sqrt{1-\tau^{-2} \nu^{-2} \xi^{2}} .
$$

The sign is such that $\pm b$ corresponds to rays with $\pm \partial z / \partial t>0$. We define a set of points $(z, x, \xi, \tau)$ associated with propagation angles with the vertical $<\theta$, by

$$
I_{\theta}^{\prime}=\left\{(z, x, \xi, \tau) \mid \tau \neq 0 \quad \text { and } \quad\left\|v(z, x)^{-1} \tau^{-1} \xi\right\| \leq \sin (\theta)\right\} .
$$

To obtain the microlocal one-way wave equations we follow the factorization argument of Taylor [8]. In this factorization it is assumed that singularities of $F$ and therefore also those of $U$ are not in the set given by $\xi=\tau=$ $0, \zeta \neq 0$. By (5) there is a constant $C$ (assuming that $v$ is bounded) such that for the propagating singularities we have

$$
|\zeta|<C|\tau|
$$

The decoupling will be done microlocally where the propagation angle $\theta$ is smaller than some fixed angle $\theta_{2}$. We define a subset of phase space $\mathbb{R}^{n+1} \times \mathbb{R}^{n+1}$ associated to such angles by

$$
I_{\theta_{2}}=\left\{(z, x, t, \zeta, \xi, \tau)\left|(z, x, \xi, \tau) \in I_{\theta_{2}}^{\prime},\right| \zeta|<C| \tau \mid\right\} .
$$

Let $I_{ \pm, \theta_{2}}$ denote the subsets of $I_{\theta_{2}}$ with $\mp \tau^{-1} \zeta>0$.

The down- and upgoing components $\left(u_{+}, u_{-}\right)$are obtained from $\left(U, \rho^{-1}(\partial U / \partial z)\right)$ by a $2 \times 2$ pseudodifferential matrix $Q=Q\left(z, x, D_{x}, D_{t}\right)$. We assume that $Q$ is elliptic, i.e. there is a matrix pseudodifferential operator $W$ such 
that $W Q \sim Q W \sim$ Id. We take the liberty to denote $Q^{-1}=W$, even though this is not quite correct. We also define sources $\left(f_{+}, f_{-}\right)$. We have

$$
\left(\begin{array}{l}
u_{+} \\
u_{-}
\end{array}\right)=Q^{-1}\left(\begin{array}{l}
U \\
\rho^{-1} \frac{\partial U}{\partial z}
\end{array}\right), \quad\left(\begin{array}{l}
f_{+} \\
f_{-}
\end{array}\right)=Q^{-1}\left(\begin{array}{l}
0 \\
F
\end{array}\right) .
$$

We have the following result about one-way wave equations for $\left(u_{+}, u_{-}\right)$. The proof is the subject of Section 3 . In the following the notation order $(k)$ stands for a contribution that is a symbol or pseudodifferential operator of order $k$.

Theorem 1. For suitably chosen $Q$ and $B_{ \pm}=B_{ \pm}\left(z, x, D_{x}, D_{t}\right)$ the equation

$$
P U \equiv F \text { microlocally on } I_{\theta_{2}}
$$

holds if and only if

$$
P_{0,+} u_{+} \stackrel{\text { def }}{=}\left(\partial_{z}-\mathrm{i} B_{+}\left(z, x, D_{x}, D_{t}\right)\right) u_{+} \equiv f_{+}, \text {microlocally on } I_{\theta_{2}},
$$

and

$$
P_{0,-} u_{-} \stackrel{\text { def }}{=}\left(\partial_{z}-\mathrm{i} B_{-}\left(z, x, D_{x}, D_{t}\right)\right) u_{-} \equiv f_{-}, \text {microlocally on } I_{\theta_{2}} .
$$

The operator $B_{ \pm}$can be chosen selfadjoint, with $B_{ \pm}$and $Q$ satisfying

$$
\begin{aligned}
& B_{ \pm}(z, x, \xi, \tau)= \pm\left(b+\frac{1}{2} \mathrm{i} b^{-1} \sum_{j=1}^{n-1} \frac{\partial b}{\partial \xi_{j}} \frac{\partial b}{\partial x_{j}}\right)+\operatorname{order}(-1) \text { on } I_{\theta_{2}}^{\prime}, \\
& Q(z, x, \xi, \tau)=\left(\begin{array}{cc}
\rho^{1 / 2} a^{-1 / 4} & \rho^{1 / 2} a^{-1 / 4} \\
i \operatorname{sgn}(\tau) \rho^{-1 / 2} a^{1 / 4} & -\mathrm{i} \operatorname{sgn}(\tau) \rho^{-1 / 2} a^{1 / 4}
\end{array}\right)+\operatorname{order}\left(\begin{array}{rr}
-\frac{3}{2} & -\frac{3}{2} \\
-\frac{1}{2} & -\frac{1}{2}
\end{array}\right) \text { on } I_{\theta_{2}}^{\prime},
\end{aligned}
$$

where $a$ is defined by $a(z, x, \xi, \tau)=v(z, x)^{2} \tau^{2}-\|\xi\|^{2}$. If we choose $Q$ such that $U=u_{+}+u_{-}$, that is $Q_{1,1}=$ $Q_{1,2}=1$, then $B_{ \pm}$satisfies

$$
B_{ \pm}(z, x, \xi, \tau)= \pm\left(b+\frac{1}{2} \mathrm{i} b^{-1} \sum_{j=1}^{n-1} \frac{\partial b}{\partial \xi_{j}} \frac{\partial b}{\partial x_{j}}\right)+\frac{1}{4} \mathrm{i} \frac{\partial a}{\partial z} a^{-1}-\frac{1}{2} \mathrm{i} \frac{\partial \rho}{\partial z} \rho^{-1}+\operatorname{order}(-1) \text { on } I_{\theta_{2}}^{\prime}
$$

The highest order term in Eq. (14) determines the Hamilton flow of the singularities, hence the rays. The zeroth-order term determines the amplitude. Thus both terms need to be incorporated for an accurate highest order approximation of the solutions using (14). There are two kinds of zeroth-order terms for $B_{ \pm}$. First the term $(1 / 2) \mathrm{i} b^{-1} \sum_{j=1}^{n-1}\left(\partial b / \partial \xi_{j}\right)\left(\partial b / \partial x_{j}\right)$. When computed explicitly it is equal to $(1 / 2) \mathrm{i} v \xi(\partial v / \partial x) \tau^{-1}\left(v^{2}-\tau^{-2} \xi^{2}\right)^{-3 / 2}$. This term makes the operator $b+(1 / 2) \mathrm{i} b^{-1} \sum_{j=1}^{n-1}\left(\partial b / \partial \xi_{j}\right)\left(\partial b / \partial x_{j}\right)$ selfadjoint up to zeroth-order. Second there is the term $(1 / 4) \mathrm{i}(\partial a / \partial z) a^{-1}-(1 / 2) \mathrm{i}(\partial \rho / \partial z) \rho^{-1}$ in $(18)$, due to the different normalization of $Q$.

Eq. (14) is only microlocal. Outside $I_{\theta_{2}}^{\prime}$ the symbol $B_{ \pm}$is not prescribed, but we choose it with real principal symbol and smooth (i.e. without the singularity of the square root). A wave front propagating on some turning ray will be propagated incorrectly with this equation. To suppress such singularities we introduce a damping term given by a pseudodifferential operator $C=C\left(z, x, D_{x}, D_{t}\right)$. The complete one-way wave equation will be of the form

$$
P_{ \pm} u_{ \pm} \stackrel{\text { def }}{=}\left(\partial_{z}-\mathrm{i} B_{ \pm}\left(z, x, D_{x}, D_{t}\right)+C\left(z, x, D_{x}, D_{t}\right)\right) u_{ \pm}=0 .
$$


It is assumed here that a solution is sought for $z>z_{0}$ (for $z<z_{0}$ the sign in front of $C\left(z, x, D_{x}, D_{t}\right)$ must be changed). We assume the dissipative term is 0 for waves propagating with angle smaller than some given angle $\theta_{1}, \theta_{1}<\theta_{2}$. The operator $C$ is also a pseudodifferential operator with homogeneous, non-negative principal symbol $c(z, x, \xi, \tau)$. We let the order be 1 , so that the length scale associated with the decay is proportional to wavelength, but this is not essential. Its main property will be

$$
\begin{aligned}
& c(z, x, \xi, \tau)=0 \quad \text { for }(z, x, \xi, \tau) \in I_{\theta_{1}}^{\prime}, \\
& c(z, x, \xi, \tau) \geq \eta\left(\xi^{2}+\tau^{2}\right)^{1 / 2} \text { for }(z, x, \xi, \tau) \text { outside } I_{\theta_{2}}^{\prime},
\end{aligned}
$$

where $\eta$ is some positive constant. In addition there is the condition that when $C=0$, then also a number of its derivatives are zero, see the precise formulation below.

We consider the approximation of solutions to the Eq. (1) with right-hand side 0, given that the solution $U$ has only singularities propagating in the + direction (or only in the - direction). So suppose that $U$ satisfies

$$
P U \equiv 0 \quad \text { for } z>z_{0},
$$

and assume it has only singularities propagating in the + direction at $z=z_{0}$, in other words

$$
\mathrm{WF}(U) \cap\left\{z=z_{0}, \tau^{-1} \zeta>0\right\}=\emptyset .
$$

We also assume that the singularities of $P U$ at $z=z_{0}$ satisfy (10) at $z=z_{0}$, which implies that the restriction $\left.U\right|_{z_{0}}$ is well defined. Let $Q_{+}=Q_{1,1}, Q_{-}=Q_{1,2}$. The approximate solution is then given by $Q_{+} u_{+}$, where $u_{+}$is the solution of

$$
\begin{aligned}
& \left.u_{+}\right|_{z_{0}}=\left.Q_{+}\left(z_{0}\right)^{-1} U\right|_{z_{0}}, \\
& P_{+} u_{+}=0 \text { for } z>z_{0} .
\end{aligned}
$$

With a point $\left(z_{0}, x, t, \xi, \tau\right) \in I_{\theta}^{\prime}$ and a time $t$ there are two associated null bicharacteristics corresponding to the two possible values of $\zeta= \pm b\left(z_{0}, x, \xi, \tau\right)$. They can be parameterized by $z$-coordinate of the ray as long as the angle of the velocity vector is smaller or equal than $\theta$. We let $\left[Z_{\min }\left(z_{0}, x, \xi, t, \tau, \theta\right), Z_{\max }\left(z_{0}, x, \xi, t, \tau, \theta\right)\right]$ be the maximal interval where this is the case, and we denote the bicharacteristic by $\gamma_{ \pm}\left(z, z_{0}, x, t, \xi, \tau\right)$. Let $c(z, x, \xi, \tau)$ be the principal symbol of $C$. It was shown in [7] that in a solution operator for (25) a pseudodifferential factor occurs with symbol

$$
\exp \left(-\int_{z_{0}}^{z} c\left(\gamma_{ \pm}\left(z^{\prime}, z, x, t, \xi, \tau\right)\right) \mathrm{d} z^{\prime}\right)
$$

This exponential is equal to 1 when the bicharacteristic $\gamma_{ \pm}$stays in $I_{\theta_{1}}$, while it is exponentially decaying to 0 for $(\xi, \tau)$ to infinity if a finite segment between $z_{0}$ and $z$ is outside the region where $c=0$. Therefore we define a subset of $\mathbb{R}^{n+1} \times \mathbb{R}^{n+1}$ that can be reached from depth $z_{0}$, while staying in $I_{\theta}$, by

$$
J_{ \pm}\left(z_{0}, \theta\right)=\left\{(z, x, t, \zeta, \xi, \tau) \mid \mp \tau^{-1} \zeta>0 \quad \text { and } \quad Z_{\min }(z, x, \xi, \tau, \theta) \leq z_{0}\right\}
$$

We will show $U$ can be approximated by $Q_{+} u_{+}$in the following way

$$
\begin{aligned}
& \mathrm{WF}\left(Q_{+} u_{+}\right) \subset \mathrm{WF}(U), \\
& Q_{+} u_{+} \equiv U \text { on } J_{+}\left(z_{0}, \theta_{1}\right), \\
& Q_{+} u_{+} \equiv 0 \text { outside } J_{+}\left(z_{0}, \theta_{2}\right) .
\end{aligned}
$$

The same is true with $u_{+}, Q_{+}$and $J_{+}$replaced by $u_{-}, Q_{-}$and $J_{-}$. 
In [7] additional assumptions on $C$ were made. We first give an example. Define a scalar function $h(y)$ that smoothly goes from constant equal to zero at $y<0$, to being positive at $y>0$ by the formula

$$
h(y)= \begin{cases}0, & y \leq 0, \\ \frac{\exp (-1 / y)}{(\exp (-1 / y)+\exp (-1 /(1-y)))}, & 0<y<1, \\ 1, & y \geq 1 .\end{cases}
$$

Now define for example

$$
C=w(z, x, \xi, \tau) h\left(v^{-1}\left\|\tau^{-1} \xi\right\|-\sin \left(\theta_{1}\right)\right),
$$

where $w$ is homogeneous of order 1 in $(\xi, \tau)$ and bounded below by some constant times $\sqrt{\tau^{2}+\xi^{2}}$.

In general we make the following assumptions. We assume that $C$ is given by a sum $C=c+C^{(1)}$, where $C^{(1)}$ is of order 0 . We will also write $C^{(0)}(z, x, \xi, \tau)=c(z, x, \xi, \tau)$. We assume that there is an integer $L>2$ such that the derivatives of order up to $L$ of $C^{(0)}$, and of order $L-2$ of $C^{(1)}$ satisfy the following bounds

$$
\begin{array}{r}
\left|\partial_{z}^{j} \partial_{x}^{\alpha} \partial_{\xi, \tau}^{\beta} C^{(k)}(z, x, \xi, \tau)\right| \leq C(1+\|(\xi, \tau)\|)^{-|\beta|-k+(j+|\alpha|+|\beta|) / L}(1+c(z, x, \xi, \tau))^{1-(j+|\alpha|+|\beta|) / L} \\
j+2 k+|\alpha|+|\beta|<L
\end{array}
$$

for some constant $C$. It was shown in [7] that (32) satisfies this property for any $L$.

The initial value problems for the operators $P_{0, \pm}$ and $P_{ \pm}$that were defined in (14) and (19) have well-defined solution operators, that we will denote by $E_{0, \pm}\left(z, z_{0}\right)$ and $E_{ \pm}\left(z, z_{0}\right)$. In [7] it was shown that these are related by a pseudodifferential operator $K_{ \pm}=K_{ \pm}\left(z, z_{0}, x, D_{x}, D_{t}\right)$ with principal symbol (26), such that $E_{ \pm} \sim K_{ \pm} E_{0, \pm}$ (with $K$ in a class of symbols more general than that given by (3)). With the assumptions on $C$ it follows that $K$ has the property

$$
\begin{aligned}
& K_{ \pm}\left(z, z_{0}, x, \xi, \tau\right) \sim 1 \text { on } J_{ \pm}\left(z_{0}, \theta_{1}\right), \\
& K_{ \pm}\left(z, z_{0}, x, \xi, \tau\right) \in S^{\infty} \text { outside } J_{ \pm}\left(z_{0}, \theta_{2}\right) \text { if } z-z_{0}>\delta
\end{aligned}
$$

if $\delta>0$ is some small constant. This results in the following theorem, that gives sufficient conditions for the approximation property of Eqs. (28)-(30) to hold. The proof is given in Section 4.

Theorem 2. Let $U=U(z, x, t)$ satisfy (22) and (23). Let $u_{+}$be the solution to (24) and (25), where $Q_{+}$, $B_{+}$are as in Theorem 1, and $C$ satisfies (20) and (33). Then there is $K$, depending on $Q_{+}, B_{+}$and $C$, satisfying (34) and (35) such that for $z>z_{0}$

$$
Q_{+} u_{+}=K U+r
$$

with $r \in C^{\infty}(] z_{0}, \infty\left[\times \mathbb{R}^{n}\right)$.

\section{Proof of Theorem 1}

The computation of $Q$ and $B_{ \pm}$is done by writing (1) as a system of first-order in $z$, and then transforming this system. Let $V$ be defined by $V=\rho^{-1}(\partial U / \partial z)$, and $A, A_{\rho}$ by

$$
\begin{aligned}
& A_{\rho}=-\rho^{-1} v(z, x)^{2} \partial_{t}^{2}+\sum_{j} \partial_{x_{j}} \rho^{-1} \partial_{x_{j}}, \\
& A=A_{1}=-v(z, x)^{2} \partial_{t}^{2}+\sum_{j} \partial_{x_{j}}^{2} .
\end{aligned}
$$


The principal symbols of these operators are $a(z, x, \xi, \tau)=v(z, x)^{2} \tau^{2}-\|\xi\|^{2}, a_{\rho}(z, x, \xi, \tau)=\rho^{-1} v(z, x)^{2} \tau^{2}-$ $\rho^{-1}\|\xi\|^{2}$. With these definitions, Eq. (1) is equivalent to the following system for the vector $(U, V)$

$$
\frac{\partial}{\partial z}\left(\begin{array}{l}
U \\
V
\end{array}\right)-\left(\begin{array}{cc}
0 & \rho \\
-A_{\rho} & 0
\end{array}\right)\left(\begin{array}{l}
U \\
V
\end{array}\right)=\left(\begin{array}{c}
0 \\
F
\end{array}\right)
$$

The transformed wave field $\left(u_{+}, u_{-}\right)$and source $\left(f_{+}, f_{-}\right)$were defined in $(12)$ from $(U, V)$ and $(0, F)$. Recall that the matrix pseudodifferential operator $Q$ is elliptic, and that $Q^{-1}$ denotes a microlocal inverse, not an exact inverse, satisfying $Q^{-1} Q \sim Q Q^{-1} \sim$ Id.

A technical complication is that the operators $Q$ and $Q^{-1}$ are not pseudodifferential operators in $(z, x, t)$ (only in $(x, t))$. We let $\psi=\psi\left(z, x, D_{z}, D_{x}, D_{t}\right)$ be a microlocal cutoff around $\xi=\tau=0$. We let its symbol $\psi(z, x, \zeta, \xi, \tau)$ be 1 for $|\zeta|<2 C|\tau|,|\zeta|>1$ and 0 for $|\zeta|>3 C|\tau|$. Eq. (13) is true if and only if

$$
\psi Q^{-1}\left[\frac{\partial}{\partial z}-\left(\begin{array}{cc}
0 & \rho \\
-A_{\rho} & 0
\end{array}\right)\right]\left(\begin{array}{c}
U \\
V
\end{array}\right) \equiv \psi Q^{-1}\left(\begin{array}{c}
0 \\
F
\end{array}\right) \text {, microlocally on } I_{\theta_{2}} .
$$

By [6, Theorem 18.1.35] the operator $\psi Q^{-1}$ is a pseudodifferential operator with symbol that equals $Q^{-1}$ modulo $S^{-\infty}$ on $I_{\theta_{2}}$. Using this theorem again we can see that in (40) a factor $Q Q^{-1}$ can be inserted in the left side before $(U, V)$. So (40) holds if and only if

$$
\psi Q^{-1}\left[\frac{\partial}{\partial z}-\left(\begin{array}{cc}
0 & \rho \\
-A_{\rho} & 0
\end{array}\right)\right] Q\left(\begin{array}{l}
u_{+} \\
u_{-}
\end{array}\right) \equiv \psi\left(\begin{array}{c}
f_{+} \\
f_{-}
\end{array}\right) \text {, microlocally on } I_{\theta_{2}} .
$$

Therefore Theorem 1 follows from the lemma that we now state concerning the diagonalization of the operator

$$
Q^{-1}\left[\frac{\partial}{\partial z}-\left(\begin{array}{cc}
0 & \rho \\
-A_{\rho} & 0
\end{array}\right)\right] Q \text {. }
$$

Lemma 3. For suitably chosen $Q$ and $B$, the operator (42) is equal to

$$
\frac{\partial}{\partial z}-\mathrm{i}\left(\begin{array}{cc}
B_{+} & 0 \\
0 & B_{-}
\end{array}\right)+R
$$

where $R=R\left(z, x, D_{x}, D_{t}\right)$ is a $2 \times 2$ matrix pseudodifferential operator of order 1 with symbol that is in $S^{-\infty}$ on $I_{\theta_{2}}^{\prime}$. Here we can choose $B_{ \pm}$selfadjoint, with $Q, B_{ \pm}$satisfying (16) and (17). We can also choose $Q$ with $Q_{1,1}=Q_{1,2}=1$, with $B_{ \pm}$satisfying (18) and $Q$ satisfying

$$
Q=\left(\begin{array}{cc}
1 & 1 \\
-\mathrm{i} \operatorname{sgn}(\tau) \rho^{-1 / 2} a_{\rho}^{1 / 2}+\operatorname{order}(0) & \mathrm{i} \operatorname{sgn}(\tau) \rho^{-1 / 2} a_{\rho}^{1 / 2}+\operatorname{order}(0)
\end{array}\right) .
$$

The proof mostly follows an argument of Taylor [8]. Some extra work is required to obtain the explicit expressions and the symmetry (selfadjointness) property. It is an order by order construction resulting in an asymptotic sum that is well defined according to standard results (see, e.g. [6, Proposition 18.1.3]). The main tool is the composition formula, which says that the product of two pseudodifferential operators $A\left(y, D_{y}\right)$ and $B\left(y, D_{y}\right)$ is again a pseudodifferential operator with symbol $A \# B$ given by the asymptotic sum

$$
\sum_{\alpha} \frac{1}{\alpha ! i^{|\alpha|}} \partial_{\eta}^{\alpha} A(y, \eta) \partial_{y}^{\alpha} B(y, \eta)
$$

Here $\alpha !=\alpha_{1} !, \ldots, \alpha_{n} !$ (if $y \in \mathbb{R}^{n}$ ).

For the computations in the proof of Lemma 3, we use pseudodifferential operators that are microlocally the square root or certain other powers of the operators $A, A_{\rho}$. In addition we use an operator $S\left(D_{t}\right)$ with symbol $-\operatorname{sgn}(\tau)$. In the following lemma we collect the needed information about these. 
Lemma 4. There is a pseudodifferential square root operator $\tilde{B}=\tilde{B}\left(z, x, D_{x}, D_{t}\right)$, satisfying $\tilde{B}^{2} \sim A$ microlocally on $I_{\theta_{2}}^{\prime}$. Its symbol satisfies

$$
\tilde{B}(z, x, \xi, \tau)=b+\frac{1}{2} \mathrm{i} b^{-1} \sum_{j=1}^{n-1} \frac{\partial b}{\partial \xi_{j}} \frac{\partial b}{\partial x_{j}}+\operatorname{order}(-1) \text { on } I_{\theta_{2}}^{\prime} .
$$

There are fourth and second roots $A_{\rho}^{s}, s=1 / 4,1 / 2$, microlocally on $I_{\theta_{2}}^{\prime}$, with principal symbol that equals $a_{\rho}(z, x, \xi, \tau)^{s}$ on $I_{\theta_{2}}^{\prime}$. There are inverse $A_{\rho}^{-s}, s=1 / 4,1 / 2,1$ microlocally on $I_{\theta_{2}}^{\tau}$, with principal symbol $a_{\rho}(z, x, \xi, \tau)^{-s}$ on $I_{\theta_{2}}^{\prime}$. The operators $\tilde{B}, A_{\rho}^{s}$ can be chosen selfadjoint. Let $S\left(z, x, D_{x}, D_{t}\right)$ be a pseudodifferential operator with symbol equal to $-\operatorname{sgn}(\tau)$ on $I_{\theta_{2}}^{\prime},|\tau|>1$, and selfadjoint. Then $\tilde{B} \sim S A^{1 / 2}$ on $I_{\theta_{2}}^{\prime}$. We have

$$
\tilde{B}=S \rho^{1 / 4} A_{\rho}^{1 / 2} \rho^{1 / 4}+\operatorname{order}(-1)=S \rho^{-1 / 4} A_{\rho}^{1 / 4} \rho A_{\rho}^{1 / 4} \rho^{-1 / 4}+\operatorname{order}(-1) \text { on } I_{\theta_{2}}^{\prime} .
$$

Proof. Following the standard argument (compare, e.g. Lemma II.6.2 in [9]) we look for the square root as an asymptotic sum $\sum_{j=0}^{\infty} T^{(j)}$ with each $T^{(j)}$ a pseudodifferential operator of order $1-j$. We let $T^{(0)}$ have principal symbol $b$, then $\left(T^{(0)}\right)^{2}=A+R^{(0)}$, with $R^{(0)}$ of order 1 . Now suppose we have $T^{(j)}, j=0, \ldots, k$, such that

$$
\left(\sum_{j=0}^{k} T^{(j)}\right)^{2}=A+R^{(k)} \text { with } R^{(k)} \text { of order } 1-k .
$$

Then we let $T^{(k+1)}$ have principal symbol $-(1 / 2) b^{-1} R^{(k)}$, and (48) is valid with $k+1$ instead of $k$ (note that this choice of $T^{(k+1)}$ is unique to highest order on $\left.I_{\theta_{2}}^{\prime}\right)$. In this case $R^{(0)}$ is $i \sum_{j=1}^{n-1}\left(\partial b / \partial \xi_{j}\right)\left(\partial b / \partial x_{j}\right)+\operatorname{order}(-1)$, which leads to (46). Because $b$ is real all the $T^{(j)}$ can be chosen selfadjoint. The square root $A_{\rho}^{1 / 2}$ follows similarly and its fourth root equals the square root of the square root. The existence of microlocal inverses is standard (see Theorem 18.1.9 in [6]).

The symbol of $S$ is locally constant on $I_{\theta_{2}}^{\prime}$ so $S$ commutes microlocally with $\rho^{s}$ and the $A_{\rho}^{s}$. Also the commutator $\left[A_{\rho}^{s}, \rho^{s^{\prime}}\right]$ is a lower order operator (of order $s / 2-1$ ). To compute the square of $S \rho^{1 / 4} A_{\rho}^{1 / 2} \rho^{1 / 4}$ we commute a factor $\rho^{1 / 4}$ to the left of the first $A_{\rho}^{1 / 2}$ and one to the right of the second $A_{\rho}^{1 / 2}$. Using that the multiple commutator is again an order lower, we find

$$
\left(S \rho^{1 / 4} A_{\rho}^{1 / 2} \rho^{1 / 4}\right)^{2}=\rho^{1 / 2} A_{\rho} \rho^{1 / 2}+\operatorname{order}(0)=A+\operatorname{order}(0) \text { on } I_{\theta_{2}}^{\prime} .
$$

Eq. (47) is clearly valid for the principal symbols. It follows from (49) and the fact that $T^{(1)}$ is unique to highest order that the first equality in (47) is satisfied. The second equality of (47) follows similarly.

Proof of Lemma 3. Commuting $Q^{-1}$ and $\partial / \partial z$ we find that (42) is equal to

$$
\frac{\partial}{\partial z}-Q^{-1}\left(\begin{array}{cc}
0 & \rho \\
-A_{\rho} & 0
\end{array}\right) Q-\frac{\partial Q^{-1}}{\partial z} Q
$$

We first consider the second term of (50), which is a contribution of order 1 (the third term is of order 0 ). The eigenvalues and eigenvectors of the principal symbol matrix

$$
\left(\begin{array}{cc}
0 & \rho \\
-a_{\rho} & 0
\end{array}\right)
$$

are given by

$$
\text { eigenvalues : } \quad \pm \mathrm{i} \rho(z, x)^{1 / 2} a_{\rho}(z, x, \xi, \tau)^{1 / 2}, \quad \text { eigenvectors : } \quad\left(\begin{array}{c}
\rho(z, x)^{1 / 2} \\
\pm \mathrm{i} a_{\rho}(z, x, \xi, \tau)^{1 / 2}
\end{array}\right) .
$$


We first make a highest order choice $Q=Q^{(0)}$ such that the matrix

$$
Q^{-1}\left(\begin{array}{cc}
0 & \rho \\
-A_{\rho} & 0
\end{array}\right) Q
$$

becomes diagonal on $I_{\theta_{2}}^{\prime}$. Below we will add lower order terms. We set

$$
Q^{(0)}=\left(\begin{array}{cc}
A_{\rho}^{-1 / 4} \rho^{1 / 4} & A_{\rho}^{-1 / 4} \rho^{1 / 4} \\
\mathrm{i} S A_{\rho}^{1 / 4} \rho^{-1 / 4} & -\mathrm{i} S A_{\rho}^{1 / 4} \rho^{-1 / 4}
\end{array}\right) \text { on } I_{\theta_{2}}^{\prime}
$$

with $S$ as in Lemma 4. Outside (9) we still require that the symbol $Q^{(0)}$ is an invertible matrix symbol of order

$$
\left(\begin{array}{cc}
-\frac{1}{2} & -\frac{1}{2} \\
\frac{1}{2} & \frac{1}{2}
\end{array}\right)
$$

It is easily seen that this is possible. The inverse of $Q^{(0)}$ satisfies

$$
Q^{(0)}(z, x, \xi, \tau)^{-1}=\frac{1}{2}\left(\begin{array}{cc}
\rho^{-1 / 4} A_{\rho}^{1 / 4} & -\mathrm{i} S \rho^{1 / 4} A_{\rho}^{-1 / 4} \\
\rho^{-1 / 4} A_{\rho}^{1 / 4} & \mathrm{i} S \rho^{1 / 4} A_{\rho}^{-1 / 4}
\end{array}\right) \text {, on } I_{\theta_{2}}^{\prime} .
$$

With this choice we find, using the equalities (47)

$$
Q^{-1}\left(\begin{array}{cc}
0 & \rho \\
-A_{\rho} & 0
\end{array}\right) Q=\left(\begin{array}{cc}
\mathrm{i} \tilde{B} & 0 \\
0 & -\mathrm{i} \tilde{B}
\end{array}\right)+\operatorname{order}(-1) \text { on } I_{\theta_{2}}^{\prime},
$$

where $\tilde{B}$ is the square root operator defined in (46).

Next we take the third term in (50). It can be seen easily that

$$
\frac{\partial A_{\rho}^{s}}{\partial z} A_{\rho}^{-s}=s \frac{\partial A_{\rho}}{\partial z} A_{\rho}^{-1}+\operatorname{order}(-1) \text { on } I_{\theta_{2}}^{\prime}
$$

It follows that

$$
\frac{\partial Q^{-1}}{\partial z} Q=\left(\begin{array}{cc}
0 & \frac{1}{4}\left(\frac{\partial A_{\rho}}{\partial z} A_{\rho}^{-1}-\frac{\partial \rho}{\partial z} \rho^{-1}\right) \\
\frac{1}{4}\left(\frac{\partial A_{\rho}}{\partial z} A_{\rho}^{-1}-\frac{\partial \rho}{\partial z} \rho^{-1}\right) & 0
\end{array}\right)+\operatorname{order}(-1) \text { on } I_{\theta_{2}}^{\prime}
$$

Thus, with $Q=Q^{(0)}$, the expression (50) is equal to

$$
\frac{\partial}{\partial z}-\mathrm{i}\left(\begin{array}{cc}
\tilde{B} & -\frac{1}{4} \mathrm{i}\left(\frac{\partial A_{\rho}}{\partial z} A_{\rho}^{-1}-\frac{\partial \rho}{\partial z} \rho^{-1}\right) \\
-\frac{1}{4} \mathrm{i}\left(\frac{\partial A_{\rho}}{\partial z} A_{\rho}^{-1}-\frac{\partial \rho}{\partial z} \rho^{-1}\right) & -\tilde{B}
\end{array}\right)+R \text { on } I_{\theta_{2}}^{\prime} .
$$

Here $R$ is a pseudodifferential operator of order 1 , that is of order -1 on the set $I_{\theta_{2}}^{\prime}$.

In expression (57) the highest, first-order part is diagonal, while there are lower order off-diagonal terms. Following [8] we will remove the off-diagonal terms order by order. To remove zeroth-order off-diagonal terms we modify $Q$, 
and set it equal to $Q=Q^{(1)}=Q^{(0)}\left(1+K^{(1)}\right)$. Here $K^{(1)}$ is an operator that remains to be chosen, is of order -1 and of the form

$$
K^{(1)}=\left(\begin{array}{cc}
0 & K_{1,2}^{(1)} \\
K_{2,1}^{(1)} & 0
\end{array}\right)
$$

This results in an additional contribution to (57) of order zero, which is given to highest, zeroth-order by

$$
\left(\begin{array}{cc}
\mathrm{i} \tilde{B} & 0 \\
0 & -\mathrm{i} \tilde{B}
\end{array}\right) K^{(1)}-K^{(1)}\left(\begin{array}{cc}
\mathrm{i} \tilde{B} & 0 \\
0 & -\mathrm{i} \tilde{B}
\end{array}\right)=\left(\begin{array}{cc}
0 & \mathrm{i}\left(\tilde{B} K_{1,2}^{(1)}+K_{1,2}^{(1)} \tilde{B}\right) \\
-\mathrm{i}\left(\tilde{B} K_{2,1}^{(1)}+K_{2,1}^{(1)} \tilde{B}\right) & 0
\end{array}\right) .
$$

It follows that there is a symbol $K^{(1)}$ such that this contribution cancels to zeroth-order off-diagonal contribution on $I_{\theta_{2}}^{\prime}$, not changing the first- and zeroth-order diagonal part. By considering further modifications of the form $Q^{(j+1)}=Q^{(j)}\left(1+K^{(j+1)}\right), K^{(j+1)}$ of order $-j$, also the lower order off-diagonal terms can be removed. This proves the existence of $B_{ \pm}$satisfying (16).

Next we prove the self adjointness. The operator $Q^{(0)}$ satisfies

$$
\left(\begin{array}{cc}
1 & 0 \\
0 & -1
\end{array}\right) Q^{(0) *}\left(\begin{array}{cc}
0 & -\mathrm{i} S \\
\mathrm{i} S & 0
\end{array}\right)=Q^{(0)-1}
$$

It follows that with $Q=Q^{(0)}$ we have

$$
Q^{-1}\left(\begin{array}{cc}
0 & \rho \\
-A_{\rho} & 0
\end{array}\right) Q=-\left(\begin{array}{cc}
1 & 0 \\
0 & -1
\end{array}\right)\left[Q^{-1}\left(\begin{array}{cc}
0 & \rho \\
-A_{\rho} & 0
\end{array}\right) Q\right]^{*}\left(\begin{array}{cc}
1 & 0 \\
0 & -1
\end{array}\right) \text {. }
$$

Hence with $Q=Q^{(0)}$ the second term (50) is the sum of an anti-selfadjoint diagonal part and a selfadjoint off-diagonal part (this also follows from explicit computation). The same property is true for $\left(\partial Q^{-1} / \partial z\right) Q$. To prove that $B_{ \pm}$can be chosen selfadjoint it is sufficient to show that this property is still true when the $Q$ is modified order by order as described above. So suppose the property holds for $Q^{(j)}$. Then there is a off-diagonal, selfadjoint $K^{(j+1)}$, with correct highest order term as above. We modify the definition of $Q^{(j+1)}$ with lower order terms according to $Q^{(j+1)}=Q^{(j)} \exp \left(K^{(j+1)}\right)$ (meaning the power series for exp). Then the microlocal inverse is given by $Q^{(j+1)-1}=\exp \left(-K^{(j+1)}\right) Q^{(j)-1}$. It is easy to see from the power series for exp that

$$
\left(\begin{array}{cc}
1 & 0 \\
0 & -1
\end{array}\right) \exp \left(-K^{(j+1)}\right)\left(\begin{array}{cc}
1 & 0 \\
0 & -1
\end{array}\right)=\exp \left(K^{(j+1)}\right) .
$$

It follows that the new matrix-valued differential operator stays the sum of an anti-selfadjoint diagonal part and a selfadjoint off-diagonal part. This shows the selfadjointness.

To compute the second choice of $Q$ and $B_{ \pm}$we replace $Q$ with $Q D$, where

$$
D=\operatorname{diag}\left(Q_{11}^{-1}, Q_{12}^{-1}\right) \text {. }
$$

If we denote the second choice of $Q$ by $\hat{Q}$, it follows easily that

$$
\hat{Q}^{-1}\left[\frac{\partial}{\partial z}-\left(\begin{array}{cc}
0 & \rho \\
-A_{\rho} & 0
\end{array}\right)\right] \hat{Q}=\frac{\partial}{\partial z}-\mathrm{i}\left(\begin{array}{cc}
B_{+} & 0 \\
0 & B_{-}
\end{array}\right)-\frac{\partial D^{-1}}{\partial z} D+R .
$$

The operator $D$ is diagonal and the principal symbol of $D$ is given by $\operatorname{diag}\left(\rho^{-1 / 2} a^{1 / 4}, \rho^{-1 / 2} a^{1 / 4}\right)$. This shows the last part of the lemma. 


\section{Proof of Theorem 2}

We prove only the + sign, the - sign proceeds in the same way. Define $v_{+}, v_{-}$by

$$
\left(\begin{array}{c}
v_{+} \\
v_{-}
\end{array}\right)=Q^{-1}\left(\begin{array}{l}
U \\
V
\end{array}\right) .
$$

Because of (23) it follows that $v_{-}\left(z_{0}\right) \equiv 0$ on $I_{\theta_{2}}^{\prime} \cap\left\{z=z_{0}\right\}$, hence

$$
v_{+}\left(z_{0}\right) \equiv Q_{+}^{-1} U\left(z_{0}\right)
$$

Since on $J_{+}\left(z_{0}, \theta_{2}\right)$ we have $-\tau^{-1} \zeta>0$, it follows that $U \equiv Q_{+} v_{+}$on $J_{+}\left(z_{0}, \theta_{2}\right)$. Eq. (22) implies that

$$
\left(\frac{\partial}{\partial z}-\mathrm{i} B_{+}\right) v_{+}=\tilde{f}
$$

for some $\tilde{f}$ satisfying $\tilde{f} \equiv 0$ on $I_{\theta_{2}}$. Let $w_{+}$be the solution to

$$
P_{0,+} w=0,\left.\quad w_{+}\right|_{z_{0}}=\left.v_{+}\right|_{z_{0}} .
$$

By the initial condition and a propagation of singularities result, and the fact that $P_{+}\left(v_{+}-w_{+}\right) \equiv 0$ on $I_{\theta_{2}}$, it follows that

$$
v_{+} \equiv w_{+}+g
$$

where $g \equiv 0$ on $J_{+}\left(z_{0}, \theta_{2}\right)$. Since the symbol of $K_{+}$is in $S^{-\infty}$ outside $J_{+}\left(z_{0}, \theta_{2}\right)$, we have $K_{+} g \equiv 0$. Hence

$$
u_{+} \equiv K_{+} w_{+} \equiv K_{+}\left(v_{+}-g\right) \equiv K_{+}\left(Q_{+}^{-1} U\right) \text {. }
$$

Therefore

$$
Q_{+} u_{+} \equiv\left(K+\left[Q_{+}, K\right] Q_{+}^{-1}\right) U .
$$

For the commutator term $\left[Q_{+}, K\right] Q_{+}^{-1}$ it follows from (34) and (35) that

$$
\begin{aligned}
& {\left[Q_{+}, K\right] Q_{+}^{-1} \sim 0 \text { on } J_{+}\left(z_{0}, \theta_{1}\right),} \\
& {\left[Q_{+}, K\right] Q_{+}^{-1} \in \mathrm{Op} S^{-\infty} \text { outside } J_{+}\left(z_{0}, \theta_{2}\right), \text { if } z-z_{0}>\delta .}
\end{aligned}
$$

This completes the proof of the theorem.

\section{References}

[1] J.F. Claerbout, Imaging the Earth's Interior, Blackwell Scientific Publications, Oxford, 1985.

[2] M.V. de Hoop, J.H. Le Rousseau, R.-S. Wu, Generalization of the phase-screen approximation for the scattering of acoustic waves, Wave Motion 31 (2000) 43-70.

[3] J.J. Duistermaat, Fourier Integral Operators, Birkhäuser, Boston, 1996.

[4] L. Halpern, L.N. Trefethen, Wide-angle one-way wave equations, J. Acoust. Soc. Am. 84 (1988) 1397-1404.

[5] L. Hörmander, The Analysis of Linear Partial Differential Operators, vol. 1, Springer-Verlag, Berlin, 1983.

[6] L. Hörmander, The Analysis of Linear Partial Differential Operators, vol. 3, Springer-Verlag, Berlin, 1985.

[7] C.C. Stolk, Parametrix for a hyperbolic initial value problem with dissipation in some region. math.AP/0312119.

[8] M.E. Taylor, Reflection of singularities of solutions to systems of differential equations, Commun. Pure Appl. Math. 28 (1975) 457-478.

[9] M.E. Taylor, Pseudodifferential Operators, Princeton University Press, Princeton, NJ, 1981. 